\title{
EDITORIAL \\ Prinzipien und Instrumente einer \\ Forschungsförderung gegen Leukämie - Tendenzen der Kind-Philipp-Stiftung
}

\author{
M. Hertl*
}

Erfolge der Forschung sind von Ideen und vom Fleiß der Wissenschaftler abhängig, zusätzlich von einer Portion Glück, und dazu gerade heute von nicht geringen finanziellen Mitteln. Forschungsförderung kann zum Anliegen jener werden, die eine tödliche Bedrohung durch Krankheiten wie Leukämie und Krebs an sich selbst oder im Familienkreis erlebten und jetzt, ohne selbst forschen zu können, darin ihre Chance sehen, sich aktiv am Kampf gegen diese Krankheiten zu beteiligen.

Philipp Reiners war in den Abendstunden des 2. Mai 1972 an Leukämie verstorben, trotz aller Therapie, die ihm die Medizin zu diesem Zeitpunkt geben konnte. Sein Vater, Dr. Walter Reiners, Inhaber einer Textilmaschinenfabrik in Mönchengladbach, rief eine Stiftung ins Leben, die für die Zukunft helfen sollte, anderen Familien dieses Leid zu ersparen. Im Stiftungsstatut heißt es: „... im Bewußtsein, daß die medizinische Wissenschaft in den Stand gesetzt werden muß, noch viel mehr als bisher in die Grundlagen der Leukämie als Krankheit und daraus in die Möglichkeiten einer nicht nur bessernden, sondern heilenden Therapie einzudringen ..."

1988 jährt sich zum 16. Mal der Todestag von Philipp. Im gleichen Jahr blickt die Kind-PhilippStiftung auf 16 Jahre fruchtbarer Tätigkeit zurück. 5,78 Mio. DM sind seitdem in die Stiftung geflossen. Für satzungsgemäße Leistungen wurden 3,7 Mio. DM ausgegeben und damit unter anderen 50 wissenschaftliche Vorhaben gefördert. Das Stiftungsvermögen beträgt zur Zeit knapp 4 Mio. DM.

Der Beirat (seine Mitglieder siehe in der untenstehenden Aufstellung) hat sich in allen Jahren immer wieder von neuem die Frage gestellt, in welcher Weise man am besten Forschung stimulieren, definierte Vorhaben fördern und die Zusammenarbeit der Wissenschaftler untereinander verbessern könne. Im Grunde spürte er mit seinen Initiativen Wege auf, die generell als Tendenz einer Forschungsförderung gelten können.

\footnotetext{
* Prof. Dr. Michael Hertl ist Vorsitzender des Beirates der Kind-Philipp-Stiftung für Leukämie-Forschung. Kinderklinik im Krankenhaus Neuwerk, Dünner Str. 214-216, D-4050 Mönchengladbach I
}

Klin. Pädiatr. 200 (1988) 155- 156

(C) 1988 F. Enke Verlag Stuttgar
1. Im Rahmen der Projektförderung werden für wissenschaftliche Arbeiten Personal- und Sachmittel zur Verfügung gestellt. Das Forschungsthema soll zur Klärung der Ursachen und zur Verbesserung der Therapie der Leukämie beitragen. In den Vergabekriterien wird die Wichtigkeit der Grundlagenforschung betont. Bevorzugt bedacht wurden Forschungsvorhaben kleineren Umfangs, die durch andere Einrichtungen nicht oder nicht rechtzeitig gefördert werden und die nun mit den eher begrenzten Mitteln einer privaten Stiftung eine Initialzündung erhalten sollen.

2. Bei den bisherigen vier Expertentagungen, die im vergangenen Jahrzehnt alle zwei Jahre stattfanden, wurden jeweils rund 20 hervorragende Wissenschaftler aus allen Teilen der Welt zur Besprechung eines eng begrenzten Spezialthema zusammengerufen, das mehr in direkter Diskussion als in vorbereiteten Vorträgen erörtert wurde.

3. Der „Kind-Philipp-Preis für LeukämieForschung" wird jedes Jahr für die beste deutschsprachige Arbeit auf dem Leukämiegebiet vergeben. Er ist inzwischen mit DM 20000,- dotiert und wird im Jahre 1988 zum 12. Mal vom Vorstand der Deutschen Arbeitsgemeinschaft für Leukämie-Forschung und -Behandlung im Kindesalter im Namen des Beirates der Stiftung verliehen.

4. Bei der personalen Forschungsförderung ging man zunächst mehr davon aus, den wissenschaftlichen Nachwuchs fördern zu wollen. So fanden in Ulm zwei Fortbildungskurse über „Probleme und Methoden der Leukämie-Forschung" mit jeweils rund 20 Teilnehmern bei einer Kursdauer von 10 Tagen statt. So wertvoll und anregend diese Kurse waren und so begeistert die Teilnehmer auf die brennenden Probleme eingingen, so hat sich letzten Endes dieses Förderungsverfahren als nicht genügend effektiv erwiesen. Nur wenige Teilnehmer dieser Kurse gingen schließlich auf Dauer in die Leukämie-Forschung. So hat man 1983 das „Kind-PhilippStipendium " geschaffen. Man will damit deutsche Forscher, die im Ausland an hervorragenden Kliniken oder Laboratorien gearbeitet haben und gern nach Deutschland zurückkehren möchten, die Rückkehr mit dem Ziel ermöglichen, daß sie ihre wissenschaftliche Arbeit an einer deutschen onkologischen Klinik nahtlos fortsetzen können. Sie erhalten für ein Jahr die Bezüge eines wissenschaftlichen Mitarbeiters mit der Auflage an die Klinik, sie für diese Zeit vom Routinebetrieb freizustellen, 
nach Ablauf der Zeit aber auf eine Planstelle zu übsrnehmen.

5. Ein "Doktorandenstipendium für єxperimentelle Leukämie-Forschung" soll jährlich an bis zu drei Doktoranden vergeben werden. Naturwissenschaftler und Mediziner können sich gleichermaßen mit einer Darstellung ihrer wissenschaftlichen Zielsetzung um ein solches Stipendium bewerben. Da davon ausgegangen wird, $\mathrm{da}$ es sich dabei um sehr ambitiöse Arbeiten handelt, sollen diese Stipendien Laufzeiten von 2 Jahren haben.

6. Es war schon von Anfang an ein Lieblingsgedanke von Dr. Reiners gewesen, Wissenschaftler, die an vergleichbaren Problemen arbeiten, an einen Tisch zu bringen, damit sie im Gespräch ihre eigenen Forschungsergebnisse auf einen Prüfstand bringen, neue Ideen aufnehmen und durch Kooperation weitierführende Einsichten erreichen. Daraus entstanden auch die Expertentagungen. Nun sollen jährlich innerhalb der Bundesrepublik die an der pädiatrisch-onkologischen Forschung interessierten Mitarbeiter der Kliniken mit Vertretern der einschlägigen Grundlagenfächer zusammengeführt werden. Sie sollen dabei in aller Ausführlichkeit ihre neuesten Forschungsergebnisse austauschen. Die Finanzierung dieser ,Jahrestagung für pädiatrisch-onkologische Forschung" übernimmt die Stiftung für zunächst 5 Jahre.

7. Innerhalb der Deutschen Arbisitsgemeinschaft für Leukämie-Forschung und -Behandlung im Kindesalter (DAL) und der Gesellschaft für Pädiatrische Onkologie (GPO) gibt es zur Zeit 10 Therapiestudien, deren Sitzungen mit ihrem jeweils hohen Zeitaufwand in einer Zusammenfassung neu organisiert werden sollen. Für diese „Studienleitertagungen der Pädiatrischen Onkclogie“ in der Bundesrepublik will die Stiftung nun zunächst für 5 Jahre die Finanzierung übernehmen. Bei diesen Studienleitertagungen werden sich auch andere Arbeitsgruppen treffen und Themen von grundsätzlicher Bedeutung für die Krankenversorgung diskutieren.

Wir haben als Menschen die leidvolle Erfahrung, daß wir der Krankheit und dem Tod ausgeliefert sind. Die „Kind-Philipp-Stiftung für Leukämie-Forschung", ebenso wie die gleichfalls in Mönchengladbach gegründete „Deutsche Leukämie-Forschungshilfe - Aktion für Krebskranke Kinder" entspringt dieser leidvollen Erfahrung. Sie steht aber auch für den Beweis, dal3 man etwas dagegensetzen kann. Beispielhaft hat dies Walter Reiners in seinem Leben mitgemacht und vorgernacht, was alles hier einen Stellenwert zur jeweils eigenen Zeit besitzt: Geduld im Ertragen, was nicht zu ändern ist, Ungeduld in der Auflehnung gegen eine scheinbar hoff- nungslose Schicksalsplage der Menschheit, Eigenes tun nach Vermögen, jenen fördern, der es noch besser kann, Vorwärtsdrängen und immer von neuem Mutmachen.

\section{Publikationen der Kind-Philipp-Stiftung:}

M. Hertl: Dr. Walter Reiners + , Klin. Pädiat. 192, (1980) 2

M. Hertl: Kind-Philipp-Stiftung für Leukämie-Forschung 1972-1982. Klin. Pådiat. 195, (1983) 143

Kind-Philipp-Stipendium. Klin. Pädiat. 196, (1984) 181

Kind-Philipp-Doktorandenstipendium für experimentelle Leukämie-Forschung. Klin. Pädiat. 199, (1987) 244

\section{Beirat der Kind-Philipp-Stiftung}

Vorsitzender

Dr.-Ing. Walter Reiners ( + )

seit 1980 Prof. Dr. Michael Hertl

weitere Mitglieder des Beirats:

der Vertreter der Stifterfamilie: Jan Reiners, Firma W. Schlafhorst \& Co., Mönchengladbach

der Vorsitzende der Deutschen Arbeitsgemeinschaft für Leukämie-Forschung und -Behandlung im Kindesalter e. V.: Prof. Dr. Günter Landbeck, Kinderklinik der Universität Hamburg; seit 1980 in seiner Nachfolge Prof. Dr. Fritz Lampert, Kinder-Poliklinik der Universität Gießen; seit 1986 in seiner Nachfolge Prof. Dr. Hansjörg Riehm, Kinderklinik der Medizinischen Hochschule Hannover

der Generalsekretär des Stifterverbandes für die Deutsche Wissenschaft: Thorwald Risler, in seiner Nachfolge seit 1979 Dr. Horst Niemeyer

der Vertreter der Deutschen Forschungsgemeinschaft: Dr. Fritz W. Fischer, in seiner Nachfolge seit 1980 Prof. Dr. Dietrich Niethammer, Kinderklinik der Universität Tübingen.

Die Kind-Philipp-Stiftung wird vom Stifterverband für die Deutsche Wissenschaft treuhänderisch verwaltet (4300 Essen 1, Postfach 230360 , Brucker Holt 56-60, Telefon: 0201/71 1051). Sonderkonto „Kind-Philipp-Stiftung für Leukämie-Forschung“" Nr. $10007 \quad 103$ beim Bankhaus Sal. Oppenheim jr. \& Cie., Köln, BLZ 37030200 . 